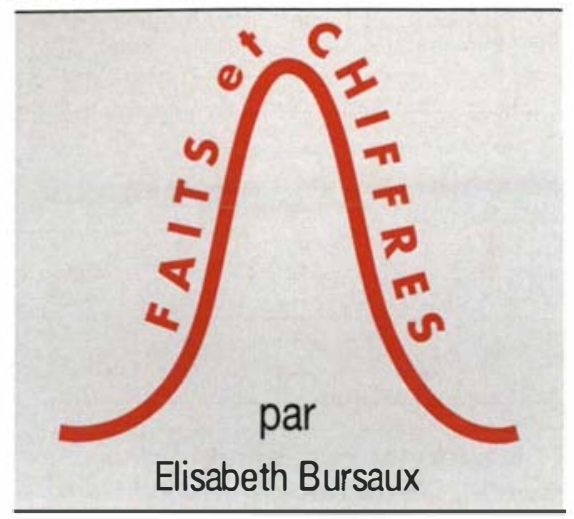

médecine/sciences $1994 ; 10: 1031-3$

\title{
Naissances répétées d'enfants souffrant de malformations congénitales
}

1. Lie RT, Wilcox AJ, Skjærven R. A population-based study of recurrence of birth defects. N EnglJ Med $1994 ; 331$ : 1-4.

2. Schulman J, Edmonds I.D, McClearn AB, Jensvold N, Shaw GM. Surveillance for and comparison of birth def ect prevalences in t o geographic areas, United States, 19831988. MMWR-CDC-Surveil Summ 1993 ; 42 : 1-7.

3. Brent RL, Gordon WE, Bennett WR, Beckman DA. Reproductive and teratologic effects of electromagnetic f elds. Reprod Toxicol $1993 ; 7: 535-80$.

4. Tuohy PG, Counsell AM, Geddis DC. The Plunket National Child Health Study: birth defects and sociodemographic factors. NZ Med J 1993 ; 106 : 489-92. es progrès de la génétique humaine (dont médecine) sciences se fait largement l'écho) et des outils diagnostiques masquent souvent le fait que la plupart des malformations congénitales n'ont pas (encore) de cause connue : est-ce dû au caractère polygénique des affections, à une interaction entre facteurs génétiques et environnementaux, ou les anomalies de développement embryonnaire et fotal surviennent-elles selon des mécanismes purement régis par le hasard ? Une importante étude norvégienne, publiée tout récemment dans le New England Journal of Medicine, aborde ce sujet [1]. Son but était de rechercher si les mères ayant donné naissance à un premier enf ant malformé couraient un risque de donner naissance à un deuxième enfant malformé supérieur à celui des mères ayant eu un premier-né normal. Il était aussi d'étudier la variation de ce risque avec le changement de partenaire sexuel et le changement d'environnement.

Il s'agit d'une étude basée sur un registre exhaustif des naissances, vivantes ou non, après 16 semaines au moins de gestation. Pour chacune des naissances, on peut connaître l'identité de la mère et (le plus souvent) celle du père. 371933 mères ont donné le jour successivement à deux enfants qui ont été inscrits au registre, de 1967 à 1989. Seules les naissances multiples ont été rejetées. Restreindre l'étude aux deux premières naissances minimise les problèmes d'interprétation de la fertilité relative qui augmentent avec les naissances successives. Les anomalies congénitales ont été décelées au cours de l'hospitalisation initiale, soit au cours des cinq premiers jours. N'ont pas été prises en compte les luxations congénitales de la hanche, à la fois très fréquentes et aux critères diagnostiques peu précis.

13607 femmes ont eu un premier enfant malformé, représentant 3,6\% des premiers-nés ; parmi celles-ci, 9192 ont eu un deuxième enfant ; elles forment le groupe cas-index pour lequel est calculé le risque d'avoir un deuxième enf ant anormal, porteur soit de la même malformation, soit d'une malformation différente (Tableau I). Les mères n'ayant pas eu de premier enfant anormal constituaient le groupe témoin. Le risque attendu pour un défaut donné est celui mesuré lors des deuxièmes naissances chez ces mères dont le premier enfant était normal. Le risque relatif pour les mères d'un premier-né malformé d'avoir un deuxième enfant anormal est le rapport entre le nombre de deuxièmes naissances anormales observées et le nombre attendu. Le risque relatif combiné pour toutes les malformations d'avoir un deuxième enfant avec la même malformation que le 


\begin{tabular}{|c|c|c|c|c|c|c|c|}
\hline \multicolumn{8}{|c|}{$\begin{array}{l}\text { RISQUES DE MALFORMATIONS CONGÉNITALES SEMBLABLES } \\
\text { OU DIFFÉRENTES CHEZ LES DEUXIĖMES ENFANTS } \\
\text { NÉS DE MĖRES D'UN PREMIER ENFANT ANORMAL }\end{array}$} \\
\hline \multirow{2}{*}{$\begin{array}{l}\text { Malformation } \\
\text { du premier bébé }\end{array}$} & \multirow[b]{2}{*}{ Nombre } & \multicolumn{6}{|c|}{ Second bébé } \\
\hline & & $\begin{array}{r}\text { Mal } \\
\text { Observées }\end{array}$ & $\begin{array}{l}\text { ormations id } \\
\text { Attendues }\end{array}$ & $\begin{array}{l}\text { ntiques } \\
\text { Risque relatif }\end{array}$ & $\begin{array}{l}\text { Malf } \\
\text { Observées }\end{array}$ & $\begin{array}{l}\text { ormations di } \\
\text { Attendues }\end{array}$ & $\begin{array}{l}\text { férentes } \\
\text { Risque relatif }\end{array}$ \\
\hline Pied-bot & 2784 & 100 & 14,7 & 7,3 & 59 & 42 & 1,4 \\
\hline Défaut génital & 1447 & 25 & 5,1 & 4,9 & 35 & 24,2 & 1,5 \\
\hline Malf. membre & 957 & 25 & 2,2 & 11,3 & 41 & 17,1 & 24 \\
\hline Malf. cardiaque & 567 & 6 & 1 & 6,0 & 11 & 10,5 & 1,1 \\
\hline Bec-de-lièvre & 436 & 18 & 0,6 & 31,4 & 10 & 8,2 & 1,2 \\
\hline Fente palatine & 144 & 3 & 0,1 & 44,5 & 2 & 2,9 & 0,7 \\
\hline Toutes ensemble & 9192 & 201 & 26,4 & 7,6 & 249 & 164,6 & 1,5 \\
\hline
\end{tabular}

Tableau II

EFFET D'UN CHANGEMENT DE MUNICIPALITÉ OU DE PARTENAIRE SUR LE RISQUE QU'UN DEUXIĖME ENFANT AIT LA MÊME MALFORMATION CONGÉNITALE QUE LE PREMIER

\begin{tabular}{|c|c|c|c|c|c|c|}
\hline \multirow[t]{3}{*}{ Partenaire } & \multirow[t]{3}{*}{ Municipalité } & \multicolumn{5}{|c|}{ Même malformation congénitale chez le deuxième bébé } \\
\hline & & \multicolumn{2}{|c|}{ Groupe cas-index } & \multicolumn{2}{|c|}{ Groupe témoin } & \multirow{2}{*}{ Risque relatif } \\
\hline & & Cas & Total & Cas & Total & \\
\hline Le même & La même & 115 & 4200 & 454 & 192990 & 11,6 \\
\hline Le même & Différente & 41 & 2332 & 310 & 89588 & 5,1 \\
\hline Différent & La même & 16 & 985 & 99 & 44196 & 7,3 \\
\hline Différent & Différente & 10 & 749 & 78 & 28861 & 4,9 \\
\hline
\end{tabular}

Le groupe cas-index est le groupe des mères dont le premier-né est malformé. Le groupe témoin est celui des mères dont le premier-né est normal. Chaque mère du groupe témoin est appariée à une mère du groupe cas-index.

premier est de 7,6. Le risque relatif varie avec la nature de la malformation ; les plus fréquemment récurrentes sont les becs-de-lièvre et les fentes palatines. Les malformations non détaillées dans le Tableau I ont eu moins de trois récurrences. En revanche, le risque relatif d'avoir un deuxième enfant présentant une malformation différente de celle du premier enfant n'est que de 1,5. Ce nombre, peu différent de 1, manifeste peut-être le plus grand soin apporté à l'examen des enfants dont l'aîné
Ce qui est passionnant dans ce travail, c'est l'étude des influences génétiques et de l'environnement dans la répétition des malformations. Des enfants avec le même père et la même mère ont la moitié de leurs gènes communs; s'ils n'ont pas le même père, ils ne partagent plus qu'un quart des gènes. Parmi les mères qui ont gardé le même partenaire, le risque relatif d'avoir un deuxième enfant porteur de la même malformation que son aîné est de 8,8 alors qu'il n'est plus que de 6,2 si le père n'est pas le même.
Mais changer de partenaire s'accompagne souvent d'un changement de vie important et les facteurs liés à l'environnement, non génétiques, tels que le régime alimentaire, le lieu de résidence, l'environnement professionnel, peuvent être très modifiés. L'effet d'un changement de résidence a été facile à étudier car l'adresse de la mère est notée sur le registre. Les résultats sont détaillés dans le Tableau II : les femmes qui sont restées avec le même partenaire sans déménager ont un risque relatif d'avoir un 
deuxième enfant avec la même anomalie que le premier de 11,6 , alors que ce risque est diminué de plus de la moitié si le couple a déménagé $(5,1)$; cette différence est très significative $(p<0,001)$; une petite baisse additionnelle, non significative, est observée si la mère a non seulement déménagé mais aussi changé de partenaire $(4,9)$. Le risque relatif d'avoir un deuxième enfant porteur d'une deuxième malformation n'est modifié ni par le changement de partenaire, ni par le changement de résidence.

Cet aspect de l'étude norvégienne est à rapprocher des résultats de l'étude américaine sur les différences de prévalence de malformations congénitales en Californie et en Géorgie [2], elle aussi basée sur l'analyse exhaustive des registres des naissances (640 000 naissances en Californie et 153000 à Atlanta) : les auteurs ne peuvent expliquer les différences de prévalence de malformations des bras et des mains par des facteurs génétiques et envisagent un rôle pour des facteurs d'environnement. On n'a pas beaucoup de pistes pour rechercher ces facteurs : les champs électromagnétiques, très à la mode pendant des années, ont montré leur innocuité tératologique [3], et même le tabac ne peut être tenu pour responsable de malformations [4]. En revanche, l'étude néozélandaise a trouvé une association significative entre le statut socio-économique et l'incidence des malformations congénitales [4].

En conclusion, des malformations congénitales spécifiques ont une forte tendance à se reproduire dans les familles, indiquant que les facteurs qui en sont responsables, sans doute purement génétiques, sont toujours à l'œuvre. Mais il y a aussi des indications fortes que l'environnement contribue au risque familial, et que d'importants facteurs tératogènes restent à découvrir. Nous sommes à la croisée des chemins en ce qui concerne la prévention des risques d'avoir des enfants souffrant d'anomalies congénitales : les outils pour les déceler seront bientôt disponibles et il pourrait alors être possible de les prévenir. Sommes-nous prêts à en payer le prix ? 